\title{
Dynamic Programming Matching with Global Features for Online Character Recognition
}

\author{
Minoru Mori*, Seiichi Uchida ${ }^{\dagger}$ and Hitoshi Sakano ${ }^{\ddagger}$ \\ ${ }^{*}$ NTT Communication Science Laboratories, NTT Corporation, Atsugi-shi, Kanagawa, 243-0198 Japan \\ E-mail: mori.minoru@lab.ntt.co.jp \\ ${ }^{\dagger}$ Faculty of Information Science and Electrical Engineering, Kyushu University, Fukuoka-shi, Fukuoka, 819-0395 Japan \\ E-mail: uchida@ait.kyushu-u.ac.jp \\ ${ }^{\ddagger}$ NTT Communication Science Laboratories, NTT Corporation, Seika-cho, Kyoto, Japan 619-0237 Japan \\ E-mail: sakano.hitoshi@lab.ntt.co.jp
}

\begin{abstract}
This paper proposes a dynamic programming (DP) matching method with global features for online character recognition. Many online character recognition methods have utilized the ability of DP matching on compensating temporal fluctuation. On the other hand, DP requires the Markovian property on its matching process. Consequently, most traditional DP matching methods have utilized local information of strokes such as $x y$-coordinates or local directions as features, because it is easy to satisfy the Markovian property with those features. Unfortunately, these local features cannot represent global structure of character shapes. Although global features that extract global structures of characters have high potential to represent various key characteristics of character shapes, conventional DP matching methods cannot handle global features. This is because the incorporation of global features is not straightforward due to the Markovian property of DP. In this paper we propose a new scheme for DP matching using global features. Our method first selects global features which not only satisfy the Markovian property but also have sufficient discrimination ability. By embedding the selected global features into DP matching process, we can compensate temporal fluctuation while considering the global structure of the pattern. Experimental results show that our methods can enhance the recognition accuracy for online numeral characters.
\end{abstract}

Keywords-DP matching, feature extraction, feature selection, global structure, online character recognition

\section{INTRODUCTION}

For online character recognition, many methods have been proposed [1]. One of major recognition methods is dynamic programming (DP) matching that is one of elastic matching techniques. DP matching methods often handle temporal sequences of local features to represent characters strokes. The $x y$-coordinates at each point on the stroke [2] or local direction [1], [3] which is derived as the relative vector of two adjacent points are mainly used as local features. This scheme has obtained good recognition accuracy. However, the locality of the characteristics in the DP matching methods with local features neglects the non-Markovian characteristics that are involved in our actual handwriting process. We, therefore, must observe only the previous point under the Markovian assumption. Thus, there is a significant risk that " 0 " will become " 6 ".

One solution to the above problem is to incorporate a global feature into the matching process. Mori et al. [4] have shown that a global feature is useful for improving the online character recognition accuracy. Their global feature is defined as the relative vector between two temporarily distant points. In other words, their global feature can describe the relationship between two temporarily distant points. For example, their global feature can represent the relationship between the first and the last points of online character pattern of " 0 ". Those points should be close to each other in order not to misrecognize as "6". For a pattern of length $I$, it is possible to consider $I(I-1) / 2$ global features. In [4], the discriminative global features are selected automatically from those $I(I-1) / 2$ features by using the AdaBoost.

Surprisingly, the conventional DP matching methods have not dealt with such a global feature. This is because the incorporation of global features is not straightforward due to the Markovian property of DP. Instead, the conventional methods have relied on local features as noted above. Therefore, if global features that satisfy the time-order and the Markovian property can be selected among all the global ones, DP matching can directly deal with global features.

In this paper, we propose a combination of DP matching scheme and global features for improving online character recognition accuracies. There are two main contributions of this paper. First, we will provide a method for selecting global features which have not only the ability to be incorporated into DP matching process but also sufficient discrimination ability. This optimal selection is also done in dynamic programing framework. Second, we will provide a new DP matching scheme where the selected global features as well as conventional local features. The proposed scheme, of course, can compensate temporal fluctuation like the original DP matching and also guarantee the global optimality of the matching result.

The rest of this paper is organized as follows: Section II 
reviews related works in feature extraction and DP matching. Section III gives a conventional DP matching algorithm with local features. Section IV explains the method to select global features and the DP matching scheme with the global features. The experiments and results are reported and discussed in Section V. Section VI derives conclusions and future work.

\section{RELATED WORKS}

\section{A. Feature for online character recognition}

As mentioned in Section I, most online character recognition methods have used $x y$-coordinate features or local direction features (e.g., [1], [3]). As the exceptions, several online character recognition methods exploit some kinds of global features. One example is the relative stroke position feature for representing inter-stroke relationship. That, unfortunately, is used as just a supplement to a local feature [5], [6]. Another example is the star feature [7] which is based on an eight-directional representation (i.e., a quantized representation) of the entire character stroke; it can be seen as a online version of the classical Sonde method [8] for offline recognition.

The trial by Izadi and Suen [9] proposed a feature, called relational context, that computes the relative pairwise distances and angles between arbitrary point pairs. Their trial, however, is merely a preliminary evaluation of the usefulness of global features. They used online patterns, each of which was re-sampled to just 6 points, and all ${ }_{6} C_{2}$ pairs were used for extracting ${ }_{6} C_{2}$ global features. Our previous research [4] has proposed the relative vector between an arbitrary point pair on the stroke and revealed that each character class has its own important global structure through the feature selection experiments.

\section{B. DP matching with several kinds of features}

Although many conventional DP matching methods for the online character recognition use $x y$-coordinate features, some DP matching-based online character recognition methods utilize both $x y$-coordinates and local directions to enhance the recognition accuracy. For example, Kobayashi et al. [10] have proposed a two-step matching technique where the directional feature is used in the first step for a rough classification and the positional feature is used in the second step.

Feature unification strategy can be found in [11]. This strategy is that the directional distance is evaluated in terms of a set of coordinate distances. Specifically, a sum of four coordinate distances was used for the matching evaluation. Two distances are for main evaluations. The other two distances are for auxiliary evaluations and used conditionally to compensate weakness of the main evaluations on several cases. Predictive DP matching method that handles two types of features in a unified manner has been proposed [12]. Predictive DP matching converts the distance of directional

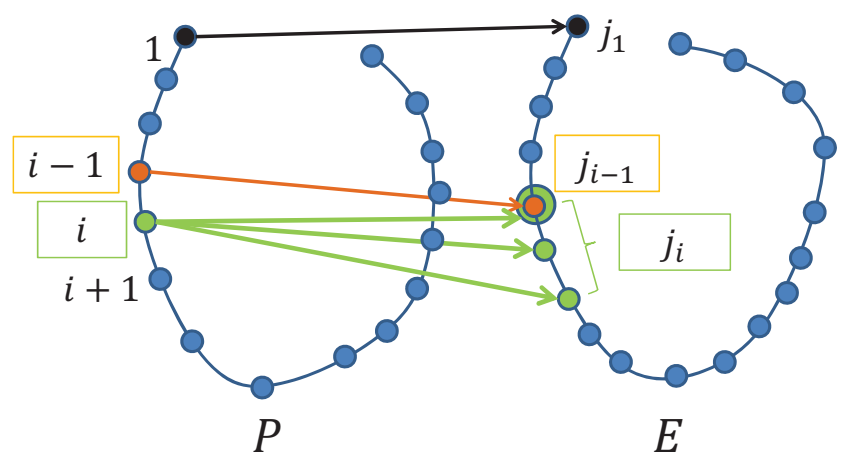

Figure 1. Conventional DP matching procedure.

features into that of coordinate features by a feature prediction technique.

These methods utilized several kind of local features but not global features. DP matching methods exploiting global features have not been proposed.

\section{CONVEntional DP MAtching With LOCAL FEATURES}

In advance to describing our proposed DP matching method with global features, this section is devoted to review the conventional DP matching with local features. Figure 1 depicts the DP procedure.

Let $E$ be an input pattern of length $J$,

$$
E=e(1), e(2), \ldots, e(j), \ldots, e(J)
$$

and $P_{c}$ be the reference pattern of length $I_{c}$ in the class $c \in[1, C]$,

$$
P_{c}=p(1), p(2), \ldots, p(i), \ldots, p(I) .
$$

For simplicity, the notations $p(i)$ and $I$ are used here instead of $p_{c}(i)$ and $I_{c}$. The vectors $p(i)$ and $e(j)$ are the following two-dimensional local feature vectors,

$$
\begin{gathered}
p(i)=(x(i), y(i)), \\
e(j)=(X(j), Y(j)),
\end{gathered}
$$

where $(x(i), y(i))$ and $(X(j), Y(j))$ are $x y$-coordinates at the each point of strokes of $P_{c}$ and $E$, respectively. Then the evaluation of the matching between $P_{c}$ and $E$ is based on the following distance

$$
d(p(i), e(j))=\|(x(i), y(i))-(X(j), Y(j))\| .
$$

The matching between $P_{c}$ and $E$ are formulated as the following optimization problem:

Minimize

$$
D=\sum_{i=1}^{I} d\left(p(i), e\left(j_{i}\right)\right)
$$


with respect to $\left\{j_{i} \mid i=1, \ldots, I\right\}$, and subject to the so-called continuity and monotonicity constraint

$$
j_{i}-j_{i-1} \in\{0,1,2\}
$$

and the boundary constraints, $j_{1}=1, j_{I}=J$. The set of variables $j_{i} \in\{1,2, \ldots, J\}$ specifies the matching (i.e., the point-to-point correspondence) between $P_{c}$ and $E$.

The above optimal matching problem has the Markovian property. That is, when we consider the multi-stage decision process of $j_{i}$ from $i=1$ to $I$, the variable $j_{i}$ depends directly on only $j_{i-1}$ and not on $j_{i-2}, \ldots, j_{1}$. Such a problem can be solved by DP.

Specifically, the DP algorithm relies on the following recursive equation:

$$
G(i, j)=d(p(i), e(j))+\min _{j^{\prime} \in\{j-2, j-1, j\}} G\left(i-1, j^{\prime}\right),
$$

where $G(i, j)$ denotes the minimum matching cost between $p(1), \ldots, p(i)$ and $e(1), \ldots, e(j)$. The DP recursion implies the fact that the minimum matching cost can be calculated recursively from $i=1$ to $I$. The cost $G(I, J)$ is the minimum distance between $P_{c}$ and $E$ and can be used as the discriminative function; the class give the minimum $G(I, J) / I$ is the class of $E$.

\section{DP Matching with Global Features}

This section describes a new scheme for DP matching exploiting global features. The proposed method consists three parts. The first part is the global feature extraction that describes the structural information of character shapes in the feature extraction stage. The second part is the alternative formulation of DP matching in order to incorporate the global features. The third part is the selection of global features for being incorporated into the above DP matching algorithm.

\section{A. Global feature extraction}

As global features, we exploit the feature proposed in [4]. This global feature is defined as a simple relative vector between an arbitrary point pair on the stroke. Details of its generation are as follows: For each of ${ }_{I} C_{2}$ point pairs, relative vector $(d x, d y)$ is calculated as the global feature. Note that the set of the ${ }_{I} C_{2}=I(I-1) / 2$ global features includes the conventional local direction features as a subset. Figure 2 depicts an example of our global feature extraction. Global features are defined and extracted between an interested point $p_{i}(i=1, \ldots, I)$ and other points for the interested point $p_{i}$.

Our global feature is simple but has high potential to describe the unique characteristics of each class. Our global features can regulate the relationship between two points widely separated on the character stroke. Especially for the online recognition task, it is possible to say that the global feature can represent the non-Markovian characteristics of handwriting.

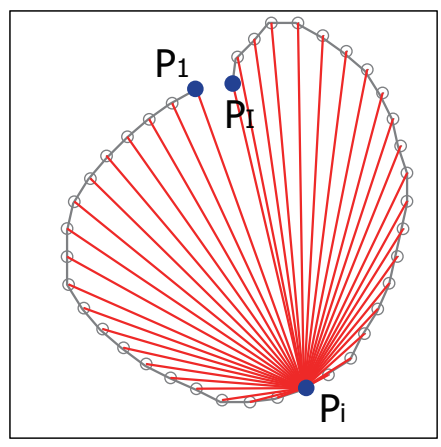

Figure 2. Global feature related to $p_{i}$.

\section{B. DP matching for global features}

The part gives the alternative formulation of DP matching to incorporate the global features. An input pattern is matched into each reference pattern using the global features in the following DP matching algorithm. The evaluation of the matching between $E$ and $P_{c}$ is based on the distance between feature vectors, $g e(l)$ and $g p(k)$. Here $g p(k)$ is the global feature, that is selected in the feature selection procedure described in section IV-C and used $k(1 \leq k, l \leq K)$ thly in DP matching, of $P_{c}$. Here $K$ is the number of the selected global features. $g e(l)$ is the selected global feature, that is used $l$-thly in matching, of $E$. Let $d_{g}(g p(k), g e(l))$ be the distance between $g p(k)$ and $g e(l)$. Then $d_{g}(g p(k), g e(l))$ is defined by

$$
\begin{aligned}
& \quad d_{g}(g p(k), g e(l)) \\
& \quad=\|(g x(k), g y(k))-(G X(l), G Y(l))\| .
\end{aligned}
$$

The matching between $P_{c}$ and $E$ are formulated as the following optimization problem:

\section{Minimize}

$$
D_{g}=\sum_{k=1}^{K} d_{g}\left(g p(k), g e\left(l_{k}\right)\right)
$$

with respect to $\left\{l_{k} \mid k=1, \ldots, K\right\}$, and subject to the socalled continuity and monotonicity constraint

$$
l_{k}-l_{k-1} \in\{0,1,2\}
$$

and the boundary constraints, $l_{1}=1, l_{K}=K$. The set of variables $l_{k} \in\{1,2, \ldots, K\}$ specifies the matching (i.e., the point-to-point correspondence) between $P_{c}$ and $E$.

Let $s(k)$ and $t(k)$ be the start point of the $k$-th global feature and the end point of that, respectively. The minimum matching cost between $g p(1), \ldots, g p(k)$ and $g e(1), \ldots, g e(k), G_{g}\left(k, s\left(l_{k}\right), t\left(l_{k}\right)\right)$, is computed by the following recursive equation: 


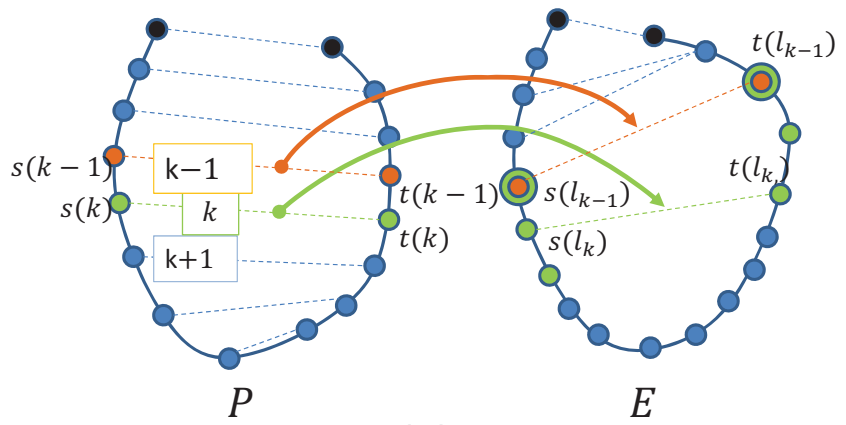

(a)

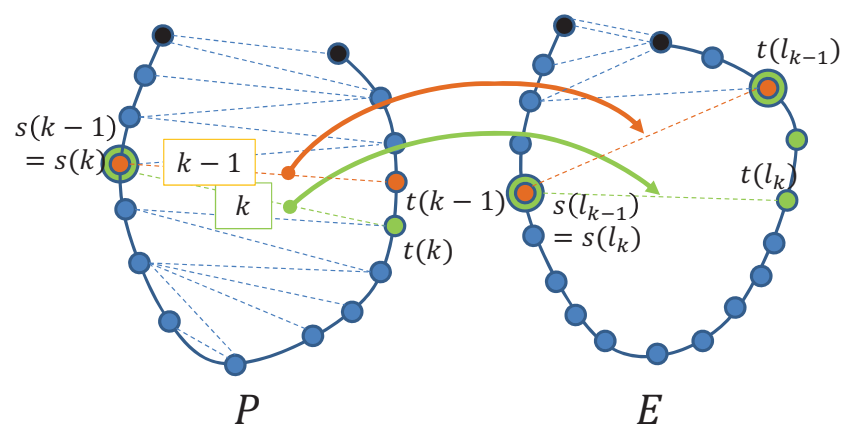

(b)

Figure 3. Procedure of global feature matching.

$$
\begin{aligned}
& G_{g}\left(k, s\left(l_{k}\right), t\left(l_{k}\right)\right)= \\
& \left\|g p(s(k), t(k))-g e\left(s\left(l_{k}\right), t\left(l_{k}\right)\right)\right\| \\
& +\left\{\begin{array}{r}
\min _{t\left(l_{k}\right)-t\left(l_{k-1}\right) \in\{0,1,2\}} G_{g}\left(k-1, s\left(l_{k}\right), t\left(l_{k-1}\right)\right) \\
\text { if } s(k)=s(k-1)
\end{array}\right. \\
& \min _{s\left(l_{k}\right)-s\left(l_{k-1}\right) \in\{0,1,2\}} G_{g}\left(k-1, s\left(l_{k-1}\right), t\left(l_{k}\right)\right) \\
& \text { if } t(k)=t(k-1) \\
& \min _{t\left(l_{k}\right)-t\left(l_{k-1}\right) \in \ldots .} G_{g}\left(k-1, s\left(l_{k-1}\right), t\left(l_{k-1}\right)\right) \\
& s\left(l_{k}\right)-s\left(l_{k-1}\right) \in \ldots
\end{aligned}
$$

Figures 3 shows the matching procedure using the global feature. For examples, Figure 3(b) corresponds to the second condition $(t(k)=t(k-1))$ in eq.(12). Although our DP matching procedure gives various matching results even under the use of global features, the feature selection method described in IV-C allows only the result (b) that only $s$ or $t$ shifts.

\section{Optimal selection of global features}

Although IV-B provides the scheme for DP matching using global features, we cannot exploit all the above $I(I-$ 1) $/ 2$ global feature in the DP-based elastic matching. The reason is that, as noted before, DP requests the Markovian property in its decision process. Therefore, we propose a feature selection method, which consider not to violate the Markovian property of the selected global features.

As the indicator for the feature efficiency, we exploit the $F$-ratio value of each feature dimension. The $F$-ratio value expresses the separability of each class on each feature dimension and are computed as follows; Let $g_{c, k}^{q}$ be the $k$-th global feature value of the $q$-th sample in the $c$-th class. The $F$-ratio value of the $k$-th global feature in the $c$-th class, $f_{c, k}$, is calculated by

$$
\begin{aligned}
& f_{c, k}=v_{c, k} / v_{k}, \\
& v_{c, k}=\sum_{q}\left(g_{c, k}^{q}-m_{c, k}\right)^{2} / N_{c}, \\
& m_{c, k}=\sum_{q} g_{c, k}^{q} / N_{c}, \\
& v_{k}=\sum_{c} \sum_{q}\left(g_{c, k}^{q}-m_{k}\right)^{2} / \sum_{c} N_{c}, \\
& m_{k}=\sum_{c} \sum_{q} g_{c, k}^{q} / \sum_{c} N_{c},
\end{aligned}
$$

where $N_{c}$ is the number of samples of the $c$-th class. $f_{c, k}$ is the simple expression of $f_{c}(s(k), t(k))$.

Next, we select global features used in the classification stage exploiting the indicator, $F$-ratio value. In this paper we regard the feature selection procedure as the optimization problem. The goal of this optimization is set to minimize the sum of the $F$-ratio values of the selected features. In other words, the $F$-ratio value is regarded as the cost for the efficient feature selection. As the optimization technique, we utilize DP matching algorithm ${ }^{1}$. The feature selection procedure is described as follows: First, the following global features satisfy the Markovian property;

$$
s(k) \geq s(k-1), t(k) \leq t(k-1) \quad \text { for all } k .
$$

To select more efficient global features to enhance the recognition accuracy, the following equations need to be satisfied;

$$
\left\{\begin{array}{l}
s(k)=s(k-1), \quad t(k)=t(k-1)-1, \text { or } \\
s(k)=s(k-1)+1, \quad t(k)=t(k-1) .
\end{array}\right.
$$

Here these conditions satisfy eq.(18).

The feature selection as the optimization problem is formulated as

Minimize

$$
H=\sum_{u=1}^{U} f_{u}=\sum_{u=1}^{U} f(s(u), t(u))
$$

where $f_{u}$ is the $F$-ratio value of the $u$-th selected global feature $(1 \leq u \leq U(=I))$. To solve this optimization, the

\footnotetext{
${ }^{1}$ The DP matching used here is apparently a different procedure from DP matching used in the classification stage
} 


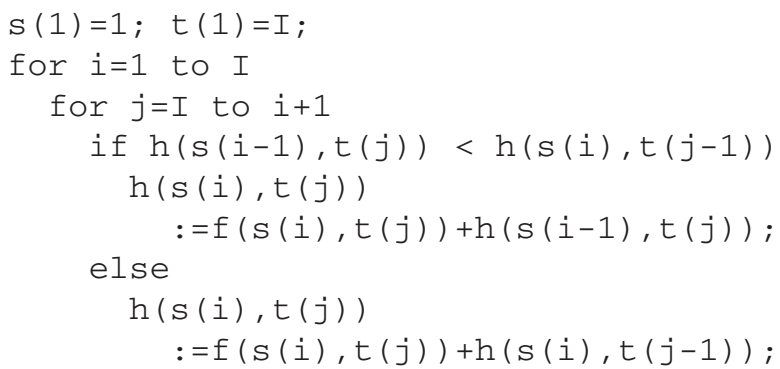

Figure 4. Procedure of recurrence formula for global feature selection.

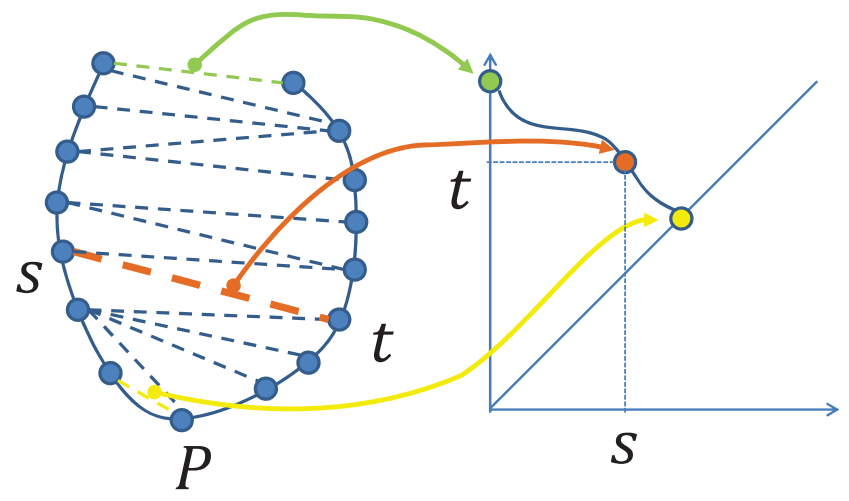

Figure 5. Feature selection procedure for global feature.

recurrence formula is defined as follows:

$$
\begin{aligned}
h(s(i), t(j)) & =f(s(i), t(j)) \\
+ & \min (h(s(i-1), t(j)), h(s(i), t(j-1)),
\end{aligned}
$$

where $h(s(i), t(j))$ is the minimum sum of the $F$-ratio values from $s(1)$ to $s(i)$ and from $t(I)$ to $t(j)$. In detail this recurrence formula is solved by the procedure shown in Figure 4.

Next, min $s(i)$ is computed by searching $i$ that provides minimum $h(s(i), s(i+1))$ and the minimum path from 1 to $\min s(i)$ is solved by the backtracking procedure. Selected global features is obtained as features involved in this minimum path. Figures 5 depicts the feature selection procedure for the global feature. Figures 6 illustrates the examples of feature selection results for the global feature. The left two examples show appropriate results that satisfy the Markovian property and obtained by our proposed algorithm. The other right one shows an inappropriate matching result because of the violation of the time-order and the Markovian property. Our proposed method can avoid such a erroneous feature selection.
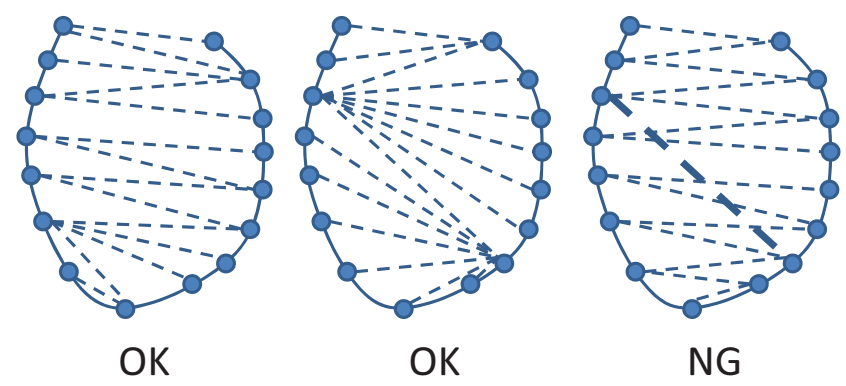

Figure 6. Examples of the selection results for the global feature.

\section{RECOGNITION EXPERIMENTS}

\section{A. Experimental setup}

Online numeral samples from the UNIPEN database [13] were used in our experiment. We selected 2,000 samples of the 10 numeral classes (“0”-“9”) for training and used another 2,000 samples of 10 classes for testing. In preprocessing, each sample was linearly normalized to $128 \times 128$ while keeping its original aspect ratio and then re-sampled at $I=40$. Finally, $I$ local features, $x y$-coordinates, and ${ }_{I} C_{2}=I(I-1) / 2$ global features were extracted from each sample.

As the discriminant function, we used the 1-Nearest Neighbor method. The input pattern is classified into the class labeled by the reference pattern that has the minimum distance between the input pattern and the interested reference pattern.

In this paper, we combine our proposed method and a conventional DP matching with $x y$-coordinate features. Let $d_{x y}\left(p(i), e\left(j_{i}\right)\right)$ be the $x y$-coordinate feature-based distance. The $d_{g}\left(g p(k), g e\left(l_{k}\right)\right)$ is the the global feature-based distance. The total sum distance $D^{\prime}$ on the combination of the both methods is formulated using a weight parameter, $\alpha$, as follows;

$$
\begin{aligned}
D^{\prime}= & \sum_{i=1}^{I} \alpha \cdot d_{g}\left(g p(k), g e\left(l_{k}\right)\right) \\
& \quad+(1-\alpha) \cdot d_{x y}\left(p(i), e\left(j_{i}\right)\right) .
\end{aligned}
$$

The minimum $D^{\prime}$ is used as the distance between the input pattern and the interested reference pattern.

\section{B. Results}

Figure 7 shows the recognition accuracies of our proposed method and conventional one for 10-class numeral classification of the test samples. The vertical axis expresses the recognition rate. The horizontal axis expresses the value of the weight parameter $\alpha$. Figure 7 provides that our proposed method has improved the recognition rate by combining both proposed and conventional methods $(94.5 \% \rightarrow 95.5 \%)$. In detailed analysis for each class, our method well improved "1" $(88 \% \rightarrow 90.5 \%)$ and " $7 "(96 \% \rightarrow 98.5 \%)$. The reason 


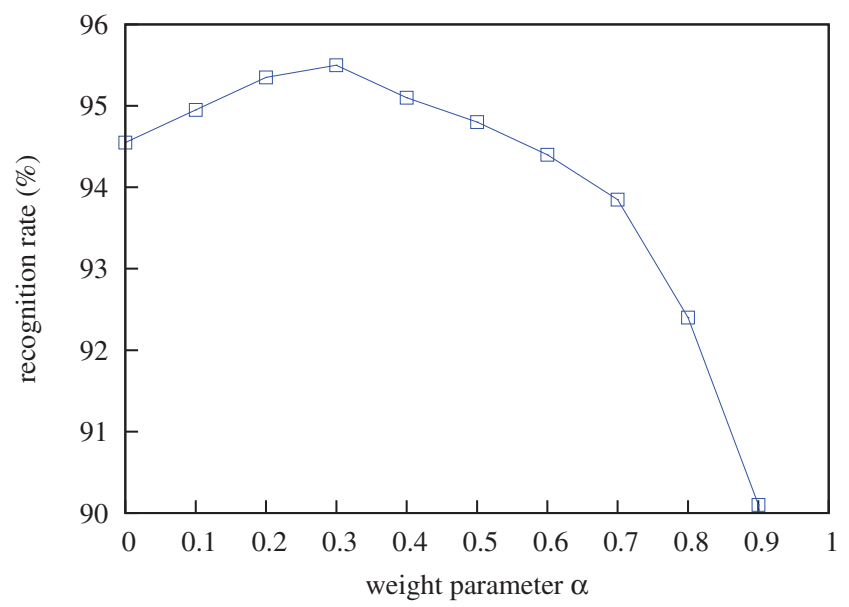

Figure 7. Recognition accuracy (vs. $\alpha$ ).

of this improvement is that the "part-to-part" correspondence of our proposed method can discriminate the difference of similar structures near the start points of " 1 " and " 7 ". On the other hand, a single use of our method or the combination that lays weight on the proposed method cannot outperform the conventional method. This reason seems that our global feature is more sensitive to stroke-order variations than the conventional $x y$-coordinate feature.

\section{CONCLUSION}

To enhance the recognition accuracy for online characters, we have proposed the DP matching method with global features. Global features are exploited for describing the global structure of a character shape. This violates the Markovian constraint that sometimes causes the erroneous point-to-point correspondence. DP matching-based feature selection method efficiently selects sets of global features to avoid the time-series disorder and inconsistency on global features using $F$-ratio values of each feature. DP matching with selected global features realizes the matching between patterns based on the "part-to-part" correspondence in the classification stage. Our experimental results using online numerical samples proved that our DP matching scheme can enhance the recognition accuracy with the cooperation of conventional DP matching with local features.

Future works are to apply our scheme to extendedDP matching method like [12], extend global features to relationship between three or multiple points and validate our method for different characters such as Latin alphabet and Chinese character.

\section{REFERENCES}

[1] C. C. Tappert, C. Y. Suen, and T. Wakahara, "The state of the art in on-line handwriting recognition," IEEE Trans. PAMI, vol. 12, no. 8, pp. 787-808, 1990.

[2] K. Ikeda, T. Yamamura, Y. Mitamura, S. Fujiwara, Y. Tominaga, and T. Kiyono, "On-line recognition of hand-written characters utilizing positional and stroke vector sequences," Pattern Recognition, vol. 13, no. 3, pp. 191-206, 1981.

[3] C. Bahlmann, "Directional feature in online handwriting recognition," Pattern Recognition, vol. 39, no. 1, pp. 115125, 2006.

[4] M. Mori, S. Uchida, and H. Sakano, "How important is global structure for characters?" in DAS'12, 2012, pp. 255-260.

[5] J.-P. Shin, M. M. Ali, Y. Katayama, and H. Sakoe, "Stroke order free on-line character recognition algorithm using interstroke information," IEICE Trans. Inf. \& Syst., vol. 82, no. 3, pp. 382-389, 1999.

[6] I. Ota, R. Yamamoto, S. Sako, and S. Sagayama, "Online handwritten kanji recognition based on inter-stroke grammar," in ICDAR'07, vol. 2, 2007, pp. 1188-1192.

[7] D. Mandalapu and S. M. Krishna, "A feature based on encoding the relative position of a point in the character for online handwritten character recognition," in ICDAR'07, vol. 2, 2007, pp. 1014-1017.

[8] R. B. Johnson, "Indicia controlled record perforating machine," U.S. Patent 2741312, 1956.

[9] S. Izadi and C. Y. Suen, "Integration of contextual information in online handwriting representation," in ICIAP'09, 2009, pp. 132-142.

[10] M. Kobayashi, S. Masaki, O. Miyamoto, Y. Nakagawa, Y. Komiya, and T. Matsumoto, "Rav (reparameterized angle variations) algorithm for online handwriting recognition," IJDAR, vol. 3, no. 3, pp. 181-191, 2001.

[11] D. Burr, "Designing a handwriting reader," IEEE Trans. PAMI, vol. 5, no. 5, pp. 554-559, 1983.

[12] D. Baba, S. Uchida, and H. Sakoe, "Predictive dp matching for on-line character recognition," in ICDAR'07, 2007, pp. 674-678.

[13] E. H. Ratzlaff, "Methods, report and survey for the comparison of diverse isolated character recognition results on the unipen database," in ICDAR'03, vol. 1, 2003, pp. 623-628. 